\title{
A Distributed Dynamic Channel Assignment and Routing Framework for Cognitive Sensor Systems
}

\author{
Celimuge $\mathrm{WU}^{\dagger \mathrm{a})}$, Satoshi OHZAHATA ${ }^{\dagger}$, Yusheng $\mathrm{JI}^{\dagger \dagger}$, and Toshihiko KATO ${ }^{\dagger}$, Members
}

\begin{abstract}
SUMMARY With the increase of the number of wireless sensing or metering devices, the collection of sensing data using wireless communication becomes an important part of a smart grid system. Cognitive radio technology can be used to facilitate the deployment of smart grid systems. In this paper, we propose a data collection and dissemination framework for cognitive radio smart grid systems to fully utilize wireless resources while maintaining a reliably connected and efficient topology for each channel. In the proposed framework, each sensor node selects a channel considering the primary user (PU) channel utilization and network connectivity. In this way, the data collection and dissemination can be performed with a high reliability and short delay while avoiding a harmful effect on primary users. We use computer simulations to evaluate the proposed framework.

key words: sensor networks, routing protocols, channel assignment, cognitive radio
\end{abstract}

\section{Introduction}

Smart grid is introduced to improve the efficiency and reliability of electricity services by using two-way information exchange between the participants and utilities. Smart grid sensors can be used to monitor weather conditions, temperature and meter devices. Wireless sensor network technology is going to act an important role in the wide spread of smart grid sensors used for monitoring and metering. With the rapid increase of the number of wireless devices, an efficient and flexible utilization of wireless resources becomes particularly critical. Since it is inefficient to allocate a dedicated channel for sensor networks, a sensor node should be intelligent to find an uncongested channel to communicate with the sink node. As a solution, cognitive radio technology has been attracting interest in recent years. Cognitive radio technology is important for facilitating the deployment of smart grid systems. However, a communication framework for cognitive radio smart grid sensing system is not discussed seriously. In this paper, we propose a joint dynamic channel assignment and routing framework which can handle data collection and dissemination in cognitive sensor systems for smart grid.

Channel assignment is an important issue for a cognitive system. There have been several channel assignment

Manuscript received January 15, 2014.

Manuscript revised June 16, 2014.

${ }^{\dagger}$ The authors are with the Department of Information Network Science, Graduate School of Information Systems, The University of Electro-Communications, Chofu-shi, 182-8585 Japan.

${ }^{\dagger \dagger}$ The author is with the Information Systems Architecture Science Research Division, National Institute of Informatics, Tokyo, 101-8430 Japan.

a) E-mail: clmg@is.uec.ac.jp

DOI: 10.1587/transinf.2013THP0012 algorithms [1]-[6]. Reference [1] requires three transceivers to handle data packets in different channels simultaneously. The use of simultaneous transmission on different channels also makes the protocol design complex and increases the channel switching incurred delay. A multi-hop communication is not considered in [2]-[6].

There have been some routing protocols for cognitive ad hoc networks [7]-[13]. However, these protocols do not consider channel assignment and switching issue. Some previous works have been done for combining dynamic channel assignment and routing [14]-[17]. Since [14]-[16] assign channels based on each source destination pair, they are not suitable for sensor applications. In [17], channel assignment is based on the established route information. This is not suitable for a sensor system in which many sensors may need to send data to a gateway at the same time.

As mentioned above, although there are several works about the channel assignment and routing issues, these works cannot provide an acceptable solution for sensor data collection and dissemination in cognitive sensor systems. The solution protocol should assign channels for sensor nodes by considering the unique features of sensor applications. The effect of MAC layer contention on the primary user (PU) performance should be considered as well. In CSMA/CA, each node does not transmit when the node senses an on-going transmission. Therefore, a secondary user should not harm the on-going transmission. Channel reassignment and rerouting problem should also be considered.

In this paper, we propose a framework as a solution for maintaining a cognitive sensor network for smart grid systems. The proposed framework, a joint Dynamic Channel Assignment and Routing (DDCAR), provides a reliable data collection and dissemination solution. Communication channels are assigned to each sensor node by using a distributed manner taking into account the channel bandwidth information and network connectivity. The channel information is obtained from upstream nodes (a gateway node or a sensor node which is nearer to the gateway than the current node). Each sensor node also creates a route (to a gateway) in the channel assignment phase. With the dynamic channel assignment, data messages can be delivered by utilizing multiple channels, which can reduce packet collisions and end-to-end delay. When the current channel cannot provide sufficient resources for sensor flows due to the arrival of new PU flows, DDCAR reassigns channels for the corresponding sensors. 
In Sect. 2, we give a brief outline of related work. In Sect. 3, we give a detailed description of the proposed framework DDCAR. Next, we present simulation results in Sect. 4. Finally, we present our conclusions and proposals for future work in Sect. 5.

\section{Related Work}

\subsection{Dynamic Channel Assignment}

There have been some proposals about dynamic channel assignment issues. Kim et al. [1] have proposed Urban$\mathrm{X}$ which uses channel and residential traffic state of external users to maximize network throughput. Since Urban$\mathrm{X}$ uses simultaneous transmission of two data transceivers in different channels, it is costly for cognitive radio sensor networks. Tan and Le [2] have investigated the channel assignment problem for cognitive radio networks with hardware-constrained SUs, and presented the optimal bruteforce search algorithm to solve the corresponding problem. Wang et al. [3] have proposed a heuristic centralized scheme to assign channels among secondary users. Li et al. [4] have proposed a residual energy aware channel assignment solution which utilizes sensor energy information and channel conditions. Yang et al. [5] have investigated the pricingbased decentralized spectrum access problem where SUs compete via random access for available spectrum opportunities. Shi et al. [6] have studied the problem of periodic sensor scheduling with limited sensor energy and communication bandwidth. However, [2]-[4] do not consider multihop communication between sensor nodes.

\subsection{Cognitive Ad Hoc Routing}

Several works have been done to solve the multi-hop routing problem. Chowdhury and Akyildiz [7] have proposed CRP, a distributed CR routing protocol for ad hoc networks. In CRP, nodes exchange route information using a control channel to capture the environmental and spectrum information. Cheng et al. [8] have proposed a spectrum aware on-demand routing by taking account of channel switching delay and backoff delay. Since the channel assignment is conducted with respect to each connection, [7], [8] are not suitable for sensor applications considered in this paper. $\mathrm{Ma}$ et al. [9] have proposed MSCRP, an on-demand routing protocol for Multi-hop Single-transceiver Cognitive Radio Networks. Cacciapuoti et al. [10] have proposed CAODV, a cognitive AODV protocol. CAODV takes advantage of the availability of multiple channels to improve the performance of AODV while considering the existence of primary users. MSCRP [9] and CAODV [10] broadcast route request messages to all available channels which increase the protocol overhead and route discovery time. Guan et al. [11] have proposed a distributed prediction-based cognitive topology control (PCTC) scheme which is a middleware-like crosslayer approach residing between $\mathrm{CR}$ modules and routing.
Liu et al. [12] have proposed an opportunistic cognitive routing protocol to improve the multi-hop transmission performance in cognitive radio networks. He et al. [13] have adopted a cross-layer approach to solve the problem of gathering correlated sensor data by employing a joint optimal design of power control and routing. However, the channel assignment and switching issue are not discussed in these protocols.

\subsection{Joint Dynamic Channel Assignment and Routing}

Ding et al. [14] have proposed ROSA, a routing and dynamic spectrum allocation algorithm for cognitive radio networks. ROSA employs a cross-layer method to maximize the network throughput by performing joint routing, dynamic spectrum allocation, scheduling, and transmit power control. Raniwala et al. [15] have proposed a multi-channel wireless mesh network architecture which includes centralized channel assignment, bandwidth allocation, and routing algorithms. Tang et al. [16] have proposed a joint routing and channel assignment (MP-JSRCA) which maximizes the network throughput by jointly selecting stable routes and avoiding inter-flow and intra-flow interferences. These protocols [14]-[16] assign channels based on each source destination pair. As a result, they are not suitable for sensor applications in which the sink node is always the traffic source or destination. Dai and Wu [17] have proposed an approach to assign channel under dynamic source routing in cognitive radio networks. Since the channel assignment is based on the established route information, it incurs frequent channel switches when the number of data flows is large.

\section{Proposed Framework}

\subsection{System Model}

Sensors (metering devices or other sensor nodes) and a gateway create a smart grid sensor system. The gateway has more than three transceivers. Here, the gateway could be an access point which has multiple antennas and a wired connection. In a real-world system, each building (or each floor of the building) could have one gateway. Each sensor node has two transceivers (control radio transceiver and data radio transceiver). There is a dedicated channel for control message exchange which we call it the control channel. Every node in the network listens for the control channel all the time using one of its transceivers (control radio transceiver). All control messages are transmitted by the control radio transceiver using the control channel. Data messages are transmitted by the data radio transceiver. Since the transmission range is limited, the messages (including both the control message and data message) could travel over multiple hops. We call a channel being used for data transmission as a working channel. Each node uses CSMA/CA to contend for a data transmission. There are multiple primary users (PUs) in the network. We consider sensor nodes 


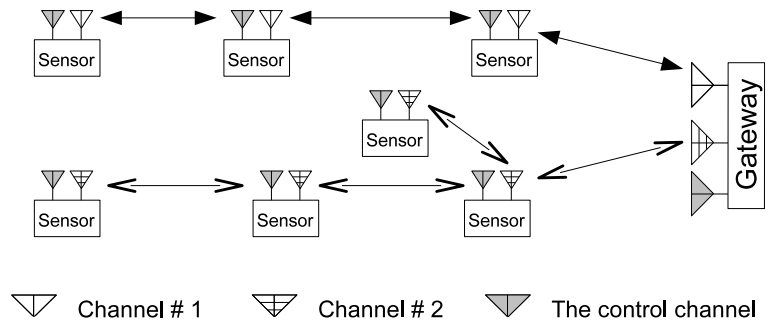

Fig. 1 Channel assignment in a smart grid system.

as secondary users (SUs). The proposed framework DDCAR aims to fully utilize wireless resources while avoiding any harmful effect on PUs. Note that the proposed protocol can be used for both licensed and unlicensed band. Sensor nodes can operate on licensed bands to achieve better performance [18]. In an unlicensed band, the users (including forwarders for a multi-hop transmission) for the on-going transmissions are considered to be PUs, and the protocol can improve the system performance as well by finding a less congested channel.

\subsection{Design Principle}

As shown in Fig. 1, in DDCAR, available channels are assigned in a distributed manner for sensors by considering channel bandwidth and connectivity of the generated network. Wireless resources are fully utilized by assigning the channel to sensor nodes efficiently. Since SU data flows (sensor flows) are distributed among multiple channels, the data collection efficiency is improved.

Channel assignment in DDCAR considers on-going data flows, and therefore a harmful effect on primary users can be avoided. When the channel bandwidth becomes not sufficient to transmit secondary user flows due to the arrival of new primary user flows or other reasons, a node chooses a different channel. The node also informs the downstream nodes (who are using itself in the data forwarding) to switch channel.

Channel assignment procedure in DDCAR can be classified to two phases, the initialization phase and maintenance phase. In the initialization phase, a simple distributed heuristic method is used to assign a channel for each sensor node. The channel assignment is triggered by a gateway. When a sensor node is directly and reliably connected with a gateway, the node can choose a channel according to the channel announcement from the gateway (see §3.5.1). Otherwise, a node chooses a channel from the channels that used by upstream nodes. Sensor nodes take account of the received signal strength in the route selection (see \$3.5.2).

In the maintenance phase, each sensor node updates the available channel information and possible routes (to the gateway) by using hello messages. In the case when a channel is getting congested due to the arrival of new primary user traffics, a sensor node can change the channel and the next hop node by using the learned information. In this way, with the cooperation among sensor nodes, the channel uti- lization is improved without any harmful effect on primary users.

\subsection{Channel Sensing and Available Bandwidth Estimation}

Channel sensing is conducted at each node with the data radio transceiver. The gateway advertises available channel list. Each sensor node also informs available channel list to its downstream sensor nodes. When the working channel is not assigned, a node senses each available channel. When a node is participating in a data transmission, the node only senses the working channel and checks whether should reassign channel or not.

In the channel sensing phase, each node calculates a channel idle time ratio (CITR). CITR is defined as $\frac{\text { idle time period }}{\text { sensing time period }}$, where sensing time period is $50 \mathrm{~ms}$. Available bandwidth of a channel at time $i$ is defined as

$$
\operatorname{LABW}(i)=\operatorname{CITR}(i) \cdot B W,
$$

where $B W$ is the overall channel bandwidth (capacity). When the node is sensing for one channel, $L A B W(i)$ is updated for each sensing time period using exponential moving average as

$$
L A B W(i) \leftarrow(1-\alpha) \cdot L A B W(i-1)+\alpha \cdot C I N R(i) \cdot B W(c),
$$

where $\alpha$ is 0.6 .

The ratio of the sensing time to transmission time is dependent on the number of available channels. When the number of available channels is large (this could possibly happen when the number of transceivers available at the gateway is large), the channel sensing time required for deciding a working channel could be large. However, this channel sensing overhead does not impair the performance of the proposed protocol because the assigned channel will be used at least until new PUs arrive.

\subsection{Link Evaluation Based on Fuzzy Logic}

Before going to describe the channel assignment and routing algorithm, we introduce the link metric used for the proposed framework. DDCAR use a fuzzy logic approach to evaluate a link based on the available bandwidth and received signal strength indication (RSSI). Available channel bandwidth and signal strength are the two important factors for evaluating a link is whether good or not. However, depending on the transmission power, multi-path fading and application requirement, the joint consideration of these two metrics can be totally different. As a result, it is difficult to use a simple metric to represent various requirements. Since fuzzy logic [19] can handle approximate reasoning which is very flexible and similar to human reasoning, it has been widely accepted in industrial communities and used in many applications. In here, we use a fuzzy metric to evaluate a wireless link. In this way, the proposed metric can be suited to all kinds of scenarios by adjusting fuzzy membership functions. In here, we use the same fuzzy reasoning 

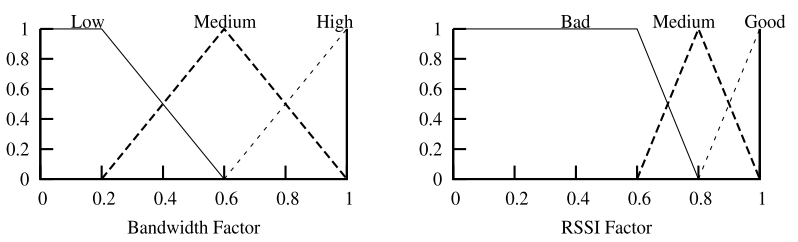

Fig. 2 Fuzzy membership functions.

Table 1 Rule base.

\begin{tabular}{l|l|l|l}
\hline & Bandwidth & Signal Strength & Rank \\
\hline \hline Rule1 & High & Good & Perfect \\
Rule2 & High & Medium & Good \\
Rule3 & High & Bad & NotAcceptable \\
Rule4 & Medium & Good & Good \\
Rule5 & Medium & Medium & Acceptable \\
Rule6 & Medium & Bad & Bad \\
Rule7 & Low & Good & NotAcceptable \\
Rule8 & Low & Medium & Bad \\
Rule9 & Low & Bad & VeryBad \\
\hline
\end{tabular}

method used in [20].

We use $F u z z(x, y)$ to show the evaluation result of the link between the node $x$ and node $y$. Bandwidth factor and RSSI factor are considered in the calculation. Bandwidth factor (BWF) (for each link and each channel) is calculated as

$$
B W F(X)=\frac{\min \left\{L A B W_{x}, L A B W_{y}\right\}}{B W},
$$

where $L A B W_{x}$ is the local available bandwidth at node $x$ and $L A B W_{y}$ is the local available bandwidth at node $y$. Upon reception a message from a neighbor $y$, node $x$ updates RSSI factor (RSSIF) as

$\operatorname{RSSIF}(x, y) \leftarrow(1-\alpha) \cdot \operatorname{RSSIF}(x, y)+\alpha \cdot\left(1-\frac{R X T h r e s h}{R x P r}\right)$.

In Eq. (4), RxPr is the received signal power, RXThresh is the reception threshold. The value of RXThresh is defined based on transceiver sensitivity. A data packet cannot be received when the received power is lower than this value.

Fuzzy membership functions defined are shown in Fig. 2. The linguistic variables of the bandwidth factor and RSSI factor are defined as $\{$ High, Medium, Low $\}$ and $\{$ Good, Medium, Bad\} respectively. DDCAR uses linguistic variables and membership functions to convert the mobility factor and RSSI factor to fuzzy values.

Fuzzy rules are defined as Table 1. Rule1 defines the following rule. IF Bandwidth is High and Signal Strength is Good THEN Rank is Perfect. A high bandwidth is required to get a high throughput. A high Signal Strength means a packet will be received by the next hop node with a high probability. This is why the Rank of the Rule1 is Perfect. Compared with the Rule1, when any one of the two factors drops to the next level, we set the Rank to be "Good" (Rule2 and Rule4). When both two factors are in the medium level, we set the rank to be "Acceptable" (Rule5). When any one of the two factors drops to the worst level, we set the Rank

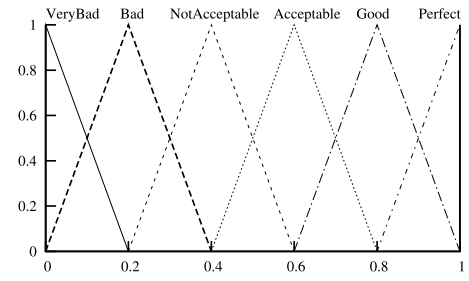

Fig. 3 Output membership function.

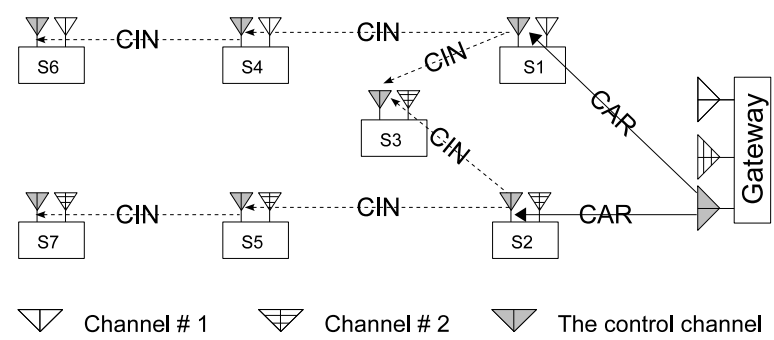

Fig. 4 Channel assignment using the control channel.

Table 2 Format of channel assignment request (CAR) message.

\begin{tabular}{|c|c|c|}
\hline \multirow{3}{*}{ GW Addr } & Channel No. 0 & $A B W(0)$ \\
\cline { 2 - 3 } & $\ldots \ldots$ & $\ldots \ldots$ \\
\cline { 2 - 3 } & Channel No. $n$ & $A B W(n)$ \\
\hline
\end{tabular}

to be "NotAcceptable" (Rule3 and Rule7). When both two factors drop to the worst level, we set the Rank to be "VeryBad" (Rule9). For other rules, we set the Rank to be "Bad" (Rule6 and Rule8). In this way, we define 9 rules in total. These rules cover all possible combinations of fuzzy values in these two factors. DDCAR uses the fuzzy values and fuzzy rules to get the rank of the link as a fuzzy value.

DDCAR uses the output membership function as defined in Fig. 3, and uses Center of Gravity (COG) [20] method to convert the fuzzy output value to $\operatorname{Fuzz}(x, y)$.

\subsection{First Round Assignment Channel in Initialization Phase}

\subsubsection{Channel Assignment Using CAR Message}

A gateway node broadcasts channel assignment request messages (CAR) using the control channel (Fig. 4). The CAR message includes the available channel information at the gateway node. The message consists of gateway address (GW Addr), channel identification (Channel No.) and available bandwidth $(A B W)$ as shown in Table 2 .

CAR is used for the selection of a working channel. In order to construct a well-connected network, a node selects a channel only when there is at least one reliable link from the gateway or an upstream node which is using the same channel. A link from the node $y$ is considered to be reliable only when

$$
\text { Fuzz }(x, y)>Q T h r e s h,
$$

where QThresh is set to 0.6 (we set this value according to 
Table 3 Format of $A C L$ maintained at each sensor node.

\begin{tabular}{|l|l|l|l|l|}
\hline \multirow{3}{*}{ GW Addr } & Channel No. 0 & $A B W(0)$ & Next hop & Metric \\
\cline { 2 - 5 } & $\ldots \ldots$ & $\ldots \ldots$ & $\ldots \ldots$ & $\ldots \ldots$ \\
\cline { 2 - 5 } & Channel No. $n$ & $A B W(n)$ & Next hop & Metric \\
\hline
\end{tabular}

Table 4 Format of channel information notification (CIN) message. \begin{tabular}{|l|l|l|l|l|}
\hline GW addr & Fwdr_list & Channel No. & $A B W$ & Metric \\
\hline
\end{tabular}

our simulation results). The optimal value of QThresh is dependent on transceiver sensitivity, noise and many other factors.

Upon reception of a CAR message, each node calculates the available channel list $(A C L)$ using the information contained in the message. The format is shown in Table 3. If a node has a reliable direct link to the gateway, the node adds all candidate channels announced by the gateway to the $A C L$ (in here we assume the link quality is symmetric). If a node has no direct reliable link to the gateway, the node adds a channel to its $A C L$ only when there is a reliable link to an upstream node which is using the same channel.

Upon reception of a CAR, CIN (see 3.5.2) or CSI (see 3.6.1) from an upstream node $y$, node $x$ updates the corresponding Metric as

$$
\operatorname{Metric}_{x}=\operatorname{Metric}_{y} \cdot \operatorname{Fuzz}(x, y) .
$$

Metric $y$ is 1 for the gateway.

Each node scans for all the channels of $A C L$, and updates the $A B W(i)$ for channel $i$ as $A B W(i)=$ $\min \{A B W(i), L A B W(i)\}$, where $L A B W$ is the sensed local available bandwidth (see Eq. 2). Based on the calculated $A B W \mathrm{~s}$, a node chooses a channel $i$ for data transmission with a probability

$$
\frac{A B W(i)}{\sum_{j \in A C L} A B W(j)} .
$$

\subsubsection{Channel Assignment Using CIN Message}

When a sensor node is not a reliable neighbor of the gateway (not a neighbor, or is a neighbor but not reliable), the node assigns itself a channel upon reception of a CIN message. The CIN message can be from a node working channel of which is already assigned. Once the data channel at a sensor node is determined, the sensor node broadcasts a channel information notification (CIN) message using the control channel. The format of the CIN message is as shown in Table 4.

As shown in Table 4, the forwarder list (Fwdr_list) consists of the nodes which forward a data message to the GW. Before sending a CIN message, each node updates the Fwdr_list by adding own address. Upon reception of a CIN message from node $y$, a node checks own address is whether in Fwdr_list or not. If own address is not in Fwdr_list, the node checks the link is reliable or not. If the link is reliable, the node updates the "Metric" field (see Table 3) of the $A C L$ as Eq. (6). The node adds or updates the corresponding
Table 5 Format of channel status information (CSI) message.

\begin{tabular}{|l|l|l|l|}
\hline GW Addr & Channel No. 0 & Metric & Fwdr_list \\
\hline
\end{tabular}

Table 6 Format of channel change indication (CCIND) message.

\begin{tabular}{|l|l|l|l|}
\hline Node Addr & Old Chann. No. & New Chann. No. & $A B W$ \\
\hline
\end{tabular}

$A C L$ entry depending on the "Metric" field. The procedure is similar to adding or updating a route entry. If the channel (indicated by the CIN message) is not in the $A C L$, the node adds a new entry to the $A C L$. If the channel is already in the $A C L$, the node checks whether the path is better (with a higher Metric) or not. If so, the node updates the "Next hop" and "Metric" field.

Since a node may receive multiple CIN messages, the node waits for a predefined time interval CIN_WAIT to collect all possible channel information. In here, we set CIN_WAIT as $20 \mathrm{~ms}$. The node then scans for the all available channel and chooses a channel based on Eq. (7). As shown in Fig. 4, sensor nodes use this procedure to select a channel and notify the assigned channel to downstream sensor nodes. The downstream sensor nodes use the same procedure to select a working channel. Once the working channel is assigned, the corresponding upstream node is used as the next hop node to the sink node (gateway). As shown in Fig. 4, node S4 uses node S1 as the next hop to the gateway.

\subsection{Maintenance Phase}

\subsubsection{Channel Status Information Sharing}

In the maintenance phase, each sensor node (including the gateway) periodically sends the channel status information (CSI) message (see Table 5) on the control channel using hello messages. The hello interval is set to 1 second. After reception of a hello message, each node updates the corresponding channel and next hop information maintained in $A C L$ as long as the Fwdr_list does not include itself and the link satisfies the QThresh constraint. In this way, each sensor node is aware of the backup channel information. Note that, for each channel, only the Fwdr_list which indicates the highest metric is maintained.

\subsubsection{PU Aware Channel Reassignment}

When a primary user is detected and channel bandwidth becomes not enough to maintain SU flows, a channel reassignment is required. A channel change indication (CCIND) message is used for channel reassignment. A CCIND message can be issued at the gateway node or any sensor node. The format of CCIND message is as shown in Table 6 . When a gateway detects a new primary user activity, the Gateway node broadcasts channel change notification (CCIND) message and current available channel list on the corresponding channel. Upon reception of a CCIND message or detection of a new PU activity, a sensor node scans 
for available channels (this information can be from the exchange of CSI messages) to find the best one. Once the new channel is detected, the node broadcasts a CCIND message on the control channel and switches to the new channel. In this way, corresponding downstream sensor nodes can switch to the new channel.

We use the following method to estimate the current channel is whether enough for handling SU flows or not. Each node senses the channel periodically. When the CITR is smaller than a predefined certain threshold CThresh, the node judges the channel is congested. In this paper, CThresh is set to 0.2 .

Once a new PU arrives and the channel becomes congested, each SU stops transmissions and scans for available channels immediately. A SU may remain at the same channel if the channel bandwidth becomes enough after some SUs switching to another channel.

\subsection{Route Selection for Sensor Nodes}

Each sensor node chooses a route to the gateway in the initialization phase. When a channel is assigned, the node uses the "Next hop" node maintained in the ACL (see Table 3) as the next forwarder node. As mentioned in mentioned in 3.5 .1 , a path is considered to be a candidate for a route only when all links of the path satisfy the QThresh constraint (see Eq. 5). If a channel assignment is triggered on the reception of a CAR message, the neighbor can directly communicate with the gateway. This means the next hop node of the route is the gateway itself. A sensor node may select a channel using the information collected from the received CIN messages (see 3.5.2) or CSI messages (see 3.6.1). In this case, the sensor node chooses the upstream node which has the minimal number of hops as the next hop.

\subsection{Loop-Free Route to the Gateway}

In DDCAR, the next hop information for a route is from the $A C L$. When a node is reliably connected with the gateway, a loop does not occur because the next hop node is the gateway itself. When a node is located at a multi-hop away from the gateway, the next hop information is updated upon reception of a CIN or CSI message. Both these two messages include Fwdr_list. Since each node determines whether to use a node as the next hop or not based on the Fwdr_list, the route being used for data forwarding is loop-free.

\subsection{Communications from Gateway to Sensors}

Since two-way communications are important for smart grid systems, we also consider communications from the gateway to sensor nodes. The communications from the gateway to sensor nodes can be multicast or unicast. In the multicast case, the gateway does not maintain a route for each sensor node. When a multicast communication is required, the gateway broadcast the data using multiple channels simultaneously. In the unicast case, after the working channel is

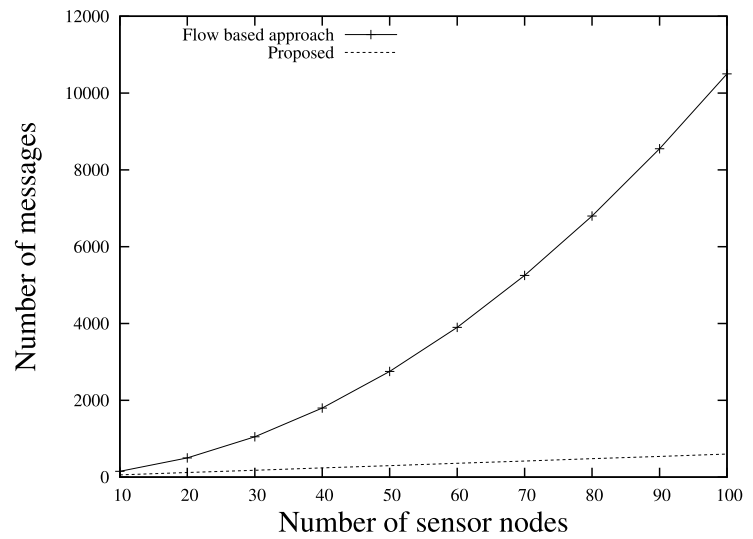

Fig. 5 Number of messages for various numbers of sensor nodes.

assigned, each sensor node sends a RREP (route reply) message to the gateway, and therefore the gateway is aware of the routes to all sensor nodes.

\subsection{Mobility Issue}

We do not consider mobility issue in the proposed framework. This is because metering devices and sensor nodes are always static. In the case when the sensor nodes are mobile, we can consider the mobility in the route selection by adding a mobility factor in the link status $(F u z z(x, y))$ estimation [20].

\subsection{Protocol Overhead}

In the proposed framework, the channel assignment is triggered from the upstream to the downstream. This makes the channel assignment can be conducted with a lower overhead as compared with the typically used traffic flow based channel assignment approach. In the flow based channel assignment approach, the channel assignment is based on the established route information, which incurs a high channel assignment overhead when the number of sensor nodes is large. In the flow based channel assignment approach, in order to find a route, route request messages are broadcasted in the network. This requires $n^{2}$ transmissions where $n$ is the number of sensor nodes. Route reply messages are transmitted from the gateway to each sensor node using unicast transmissions. Therefore, the number of messages generated is

$$
N_{m}=n^{2}+\sum_{i=1}^{n} N_{h}(i)
$$

where $N_{h}(i)$ is the number of hops to the gateway (for node $i$ ). In the proposed framework, the number is

$$
N_{m}^{\prime}=n+\sum_{i=1}^{n} N_{h}(i) .
$$

Figure 5 shows the comparison of the number of messages between the traditional approach and the proposed approach 
assuming the average number of hops is 5 (note that the node density is the dominant factor for the overhead). The figure shows that the proposed framework can significantly reduce the message overhead especially when the number of sensor nodes is large.

\section{Simulation Results}

Network Simulator 2 (ns-2.31) [21] was used to conduct simulations. We used a $500 \mathrm{~m} \times 120 \mathrm{~m}$ rectangle area. Sensor nodes were randomly distributed, and the gateway was located at the rightmost center. Simulation parameters are shown in Table 7 . Packet size was 512 bytes. The simulation time was $300 \mathrm{~s}$. We launched simulations with 50 different node deployments, and analyzed the average value of the results. The total number of nodes was 50. There were 3 random primary user (PU) flows (each with 300 kbps) of which QoS should be guaranteed. Here, a PU flow (which could be a multi-hop flow) is a traffic flow which has a higher priority than other flows. In real-world applications, the priority is represented by a higher QoS requirement or an authorized access to the corresponding frequency (or channel). Primary users are the nodes which are participating in a data forwarding of a primary traffic flow. One PU flow started at time 0.1 seconds and other two started at time 10 seconds. This is to evaluate the dynamic channel assignment mechanism of DDCAR. Sensor nodes were considered as secondary users (SUs). Each sensor node sent data to the gateway periodically with a random start time between 0 second to 5 seconds. Channel capacity was 11 Mbps. The gateway node had three transceivers and sensor nodes had two transceivers. The number of available channels was 2 . For the gateway, the three transceivers were assigned for the control channel, channel No. 1, and channel No. 2 respectively. All PU flows were transmitted on channel No. 1. We used the Nakagami propagation model. The parameters of the Nakagami model are shown in Table 8. We believe these parameters can simulate a realistic fading environment. Other simulation parameters were the default settings of ns-2.31.

In $\$ 3.11$, we explained that the flow based approach is not suitable for sensor network applications considered

Table 7 Simulation environment.

\begin{tabular}{l|l}
\hline Topology & $500 \mathrm{~m} \times 120 \mathrm{~m}$ \\
\hline Number of nodes & 50 \\
\hline Topology generation & Randomly deployed (static) \\
\hline Sensor Data & CBR, Packet size: 512 bytes \\
\hline MAC & IEEE 802.11 MAC $(11 \mathrm{Mbps})$ \\
\hline Propagation model & Nakagami Model \\
\hline Simulation time & $300 \mathrm{~s}$ \\
\hline
\end{tabular}

Table 8 Parameters of Nakagami model.

\begin{tabular}{l|l|l|l|l}
\hline gamma0_- & gamma1 $_{-}$ & gamma2 $_{-}$ & $\mathrm{d} 0_{-}$gamma & d1_gamma \\
\hline \hline 1.8 & 2.0 & 2.0 & 90 & 110 \\
\hline $\mathrm{m} 0_{-}$ & $\mathrm{m} 1_{-}$ & $\mathrm{m} 2_{-}$ & $\mathrm{d} 0_{-} \mathrm{m}_{-}$ & $\mathrm{d} 1_{-} \mathrm{m}_{-}$ \\
\hline \hline 1.1 & 1.05 & 1 & 80 & 100
\end{tabular}

in this paper. Therefore, we compared DDCAR with other two possible alternatives, One-Chann and Rnd-Chann. In One-Chann, all sensor nodes use the same channel. In RndChann, each node randomly selects a channel for data forwarding. In the following simulation results, the error bars indicate the $95 \%$ confidence intervals.

\subsection{Packet Delivery Ratio for Various Sensor Data Rates}

In each simulation scenario, every sensor node was sending data to the gateway using the same data rate. Figure 6 shows packet delivery ratio of sensor traffics (SUs) for various sensor data rates. We calculate this performance metric as the number of messages generated divided by the number of data packets received by the gateway node. As shown in Fig. 6, DCAR can efficiently utilize wireless resources by using the dynamic channel assignment mechanism, resulting in the highest packet delivery ratio. When the data rate increases to $12 \mathrm{kbps}$, One-Chann drops many packets. This shows that the use of a fixed channel for all nodes is a bad choice when the data rate is high. Due to the random channel assignment, Rnd-Chann works better than the One-Chann. However, Rnd-Chann fails to work when the data rate increases further because the protocol does not consider the existing flows (channel usage) in the channel assignment.

Figure 7 shows the packet delivery ratio for PUs. DCAR does not incur any negative effect on the PU packet delivery ratio. One-Chann and Rnd-Chann show harmful effects on the packet delivery ratio of PUs when the data rate is higher than $12 \mathrm{kbps}$.

\subsection{End-to-End Delay for Various Sensor Data Rates}

Figure 8 shows end-to-end delay for various sensor data rates. Since all sensor nodes are using the same channel, One-Chann shows a high end-to-end delay due to the increased MAC layer contention time at each node. RndChann does not provide a satisfactory result because the approach cannot balance the traffics among different channels.

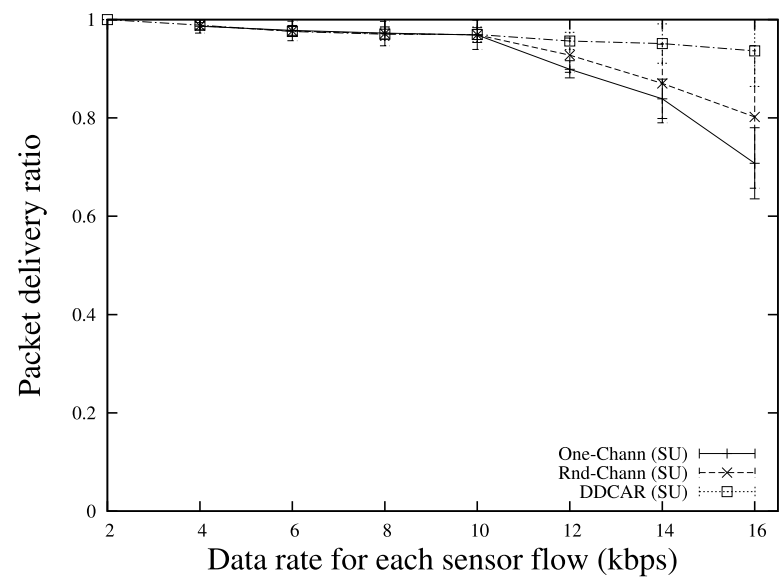

Fig. 6 PDR of SUs for various sensor data rates. 


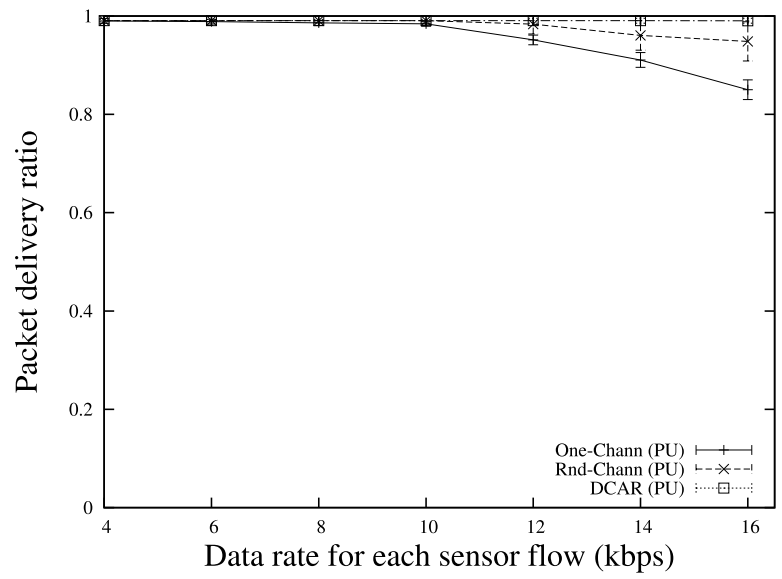

Fig. 7 PDR of PUs for various sensor data rates.

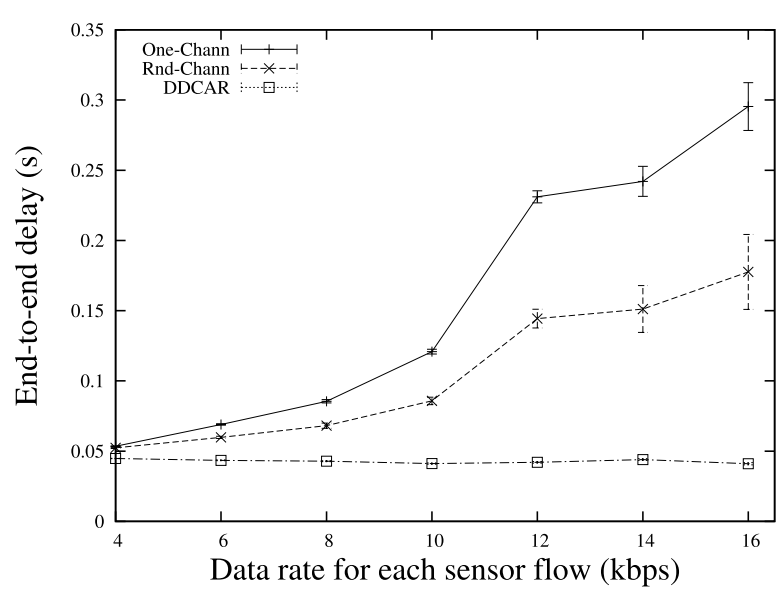

Fig. 8 End-to-end delay for various sensor data rates.

DDCAR shows the lowest end-to-end delay with the full utilization of wireless resources. This significant advantage over other two protocols shows the efficiency of the channel assignment mechanism in DDCAR.

\subsection{Performance for Broadcast Traffics}

Since two-way communications are important for a smart grid system, the packet dissemination ratio from the gateway to sensor nodes also should be considered. Broadcast is the most efficient way to announce new information to all sensors. Broadcast is important in communication between the gateway and a sensor node in a data-centric routing scenario [22], [23]. Therefore, we evaluated the dissemination ratio of broadcast traffics. In order to clarify the performance of the broadcast traffics, other data traffics were not generated.

Figure 9 shows the packet dissemination ratio for various numbers of broadcast flows. Each broadcast flow was sending 1 packet per second. This is plausible because broadcast is used for many protocols. Without loss of generality, we used Flooding for the data broadcast. In Flooding, every node rebroadcasts a packet upon the first reception. In

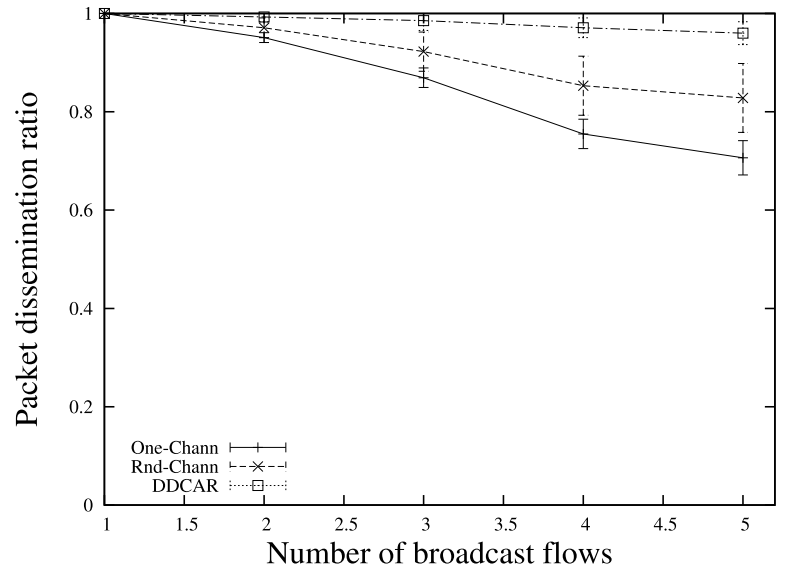

Fig. 9 Packet delivery ratio for various numbers of broadcast flows (each with 1 packet per second data rate).

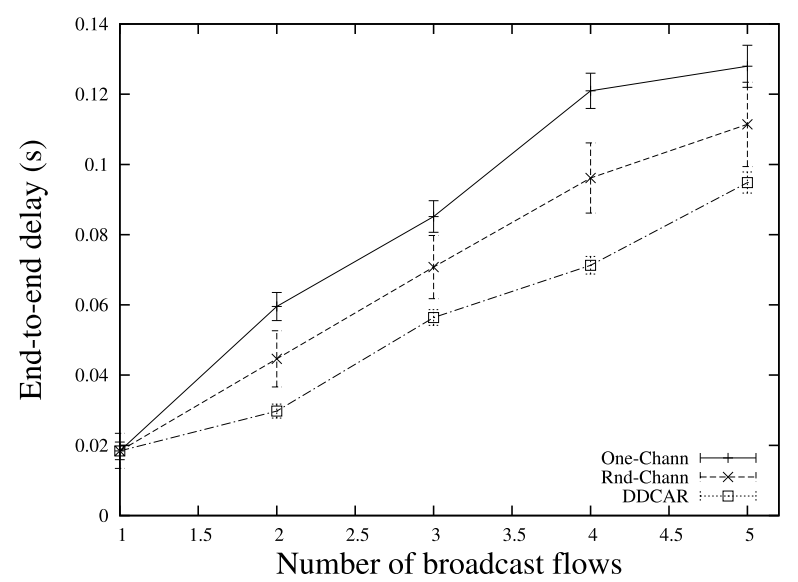

Fig. 10 End-to-end delay for various numbers of broadcast flows (each with 1 packet per second data rate).

this way, a data message can be disseminated to a multi-hop distance. One-Chann uses only one channel for communication. As a result, when the number of broadcast traffics is large, One-Chann shows a very low packet dissemination ratio due to packet collisions. Rnd-Chann does not consider network connectivity issue in the channel assignment. This results in a weakly connected (unreliable link due to channel fading) network topology, which is a main reason for packet losses. This is because there is no acknowledgement for a broadcast packet in IEEE 802.11 MAC. DDCAR assigns channels for sensor nodes by considering the network connectivity issue. The data messages can be disseminated using multiple channels and therefore data collisions are avoided.

Figure 10 shows the end-to-end delay for various numbers of broadcast flows. DDCAR can significantly reduce the end-to-end delay with its efficient channel assignment. This result shows that an efficient channel assignment is very important for dissemination of broadcast messages which are widely used in many sensor applications. 


\section{Conclusions and Future Works}

We have proposed DDCAR, a distributed dynamic channel assignment and routing framework for cognitive radio smart grid systems. DDCAR can efficiently assign available channels by considering the primary user privileges and network connectivity. Each sensor node constructs a reliable route to the gateway for the sensing data forwarding in the channel assignment phase. A channel reassignment is triggered when the working channel is congested. DDCAR also considers the communication from a gateway to sensor nodes, and uses a multi-hop broadcast or unicast communication depending on the number of receivers. We used simulations to evaluate the performance of DDCAR. DDCAR have shown a high performance in terms of packet delivery ratio and end-to-end delay with its efficient channel assignment and routing scheme.

The parameters used in this paper are defined based on our simulation results. In the near future, we will tune these parameters by doing experiments on real devices. We will consider the sensor mobility and PU mobility in the future design.

\section{References}

[1] W. Kim, A.J. Kassler, M.D. Felice, and M. Gerla, "Urban-X: Towards distributed channel assignment in cognitive multi-radio mesh networks," Proc. IFIP Wireless Days, pp.1-5, 2010.

[2] L.T. Tan, and L.B. Le, "Channel assignment with access contention resolution for cognitive radio networks," IEEE Trans. Veh. Technol., vol.61, no.6, pp.2808-2823, 2012.

[3] W. Wang, B. Kasiri, C. Jun, and A.S. Alfa, "Channel assignment of cooperative spectrum sensing in multi-channel cognitive radio networks," Proc. IEEE ICC, pp.1-5, 2011.

[4] X. Li, D. Wang, J. McNair, and J. Chen, "Residual energy aware channel assignment in cognitive radio sensor networks," Proc. IEEE WCNC, pp.398-403, 2011.

[5] L. Yang, H. Kim, J. Zhang, M. Chiang, and C.W. Tan, "Pricingbased decentralized spectrum access control in cognitive radio networks,” IEEE Trans. Netw., vol.21, no.2, pp.522-535, 2013.

[6] L. Shi, P. Cheng, and J. Chen, "Optimal periodic sensor scheduling with limited resources," IEEE Trans. Autom. Control, vol.56, no.9, pp.2190-2195, 2011.

[7] K.R. Chowdhury and I.F. Akyildiz, "CRP: A routing protocol for cognitive radio ad hoc networks," IEEE J. Sel. Areas Commun., vol.29, no.4, pp.794-804, 2011.

[8] G. Cheng, W. Liu, Y. Li, and W. Cheng, "Spectrum aware ondemand routing in cognitive radio networks," Proc. IEEE DySPAN, pp.571-574, 2007

[9] H. Ma, L. Zheng, X. Ma, and Y. luo, "Spectrum aware routing for multi-hop cognitive radio networks with a single transceiver," Proc. CrownCom, pp.1-6, 2008

[10] A.S. Cacciapuoti, C. Calcagno, M. Caleffi, and L. Paura, "CAODV: Routing in mobile ad-hoc cognitive radio networks," Proc. IFIP Wireless Days, pp.1-5, 2010.

[11] Q. Guan, F.R. Yu, S. Jiang, and G. Wei, "Prediction-based topology control and routing in cognitive radio mobile ad hoc networks," IEEE Trans. Veh. Technol., vol.59, no.9, pp.4443-4452, 2010.

[12] Y. Liu, L.X. Cai, and X. Shen, "Spectrum-aware opportunistic routing in multi-hop cognitive radio networks," IEEE J. Sel. Areas Commun., vol.30, no.10, pp.1958-1968, 2012.

[13] S. He, J. Chen, D.K.Y. Yau, and Y. Sun, "Cross-layer optimization of correlated data gathering in wireless sensor networks," IEEE Trans. Mobile Comput., vol.11, no.11, pp.1678-1691, 2012.

[14] L. Ding, T. Melodia, S.N. Batalama, J.D. Matyjas, and M.J. Medley, "Cross-layer routing and dynamic spectrum allocation in cognitive radio ad hoc networks," IEEE Trans. Veh. Technol., vol.59, no.4, pp.1969-1979, 2010.

[15] A. Raniwala, K. Gopalan, and T. Chiueh, "Centralized channel assignment and routing algorithms for multi-channel wireless mesh networks," ACM Mobile Computing and Communications Review, vol.8, pp.50-65, 2004.

[16] F. Tang, L. Barolli, and J. Li, "A joint design for distributed stable routing and channel assignment over multi-hop and multi-flow mobile ad hoc cognitive networks," IEEE Trans. Ind. Informatics, doi:10.1109/TII.2012.2228873, 2013.

[17] Y. Dai and J. Wu, "Efficient channel assignment under dynamic source routing in cognitive radio networks," Proc. IEEE MASS, pp.550-559, 2011.

[18] O.B. Akan, O. Karli, and O. Ergul, "Cognitive radio sensor networks," IEEE Netw., vol.23, no.4, pp.34-40, 2009.

[19] G.J. Klir, Ute St. Clair, and Y. Bo, Fuzzy set theory: Foundations and applications, Prentice-Hall, 1997.

[20] C. Wu, S. Ohzahata, and T. Kato, "VANET broadcast protocol based on fuzzy logic and lightweight retransmission mechanism," IEICE Trans. Commun., vol.E95-B, no.2, pp.415-425, Feb. 2012.

[21] The Network Simulator - ns-2, http://www.isi.edu/nsnam/ns/, Accessed on June 23, 2011.

[22] C. Intanagonwiwat, R. Govindan, and D. Estrin, "Directed diffusion: A scalable and robust communication paradigm for sensor networks," Proc. ACM MobiCOM, pp.56-67, 2000.

[23] J. Heidemann, F. Silva, and D. Estrin, "Matching data dissemination algorithms to application requirements," Proc. ACM SenSys, pp.218-229, 2003.

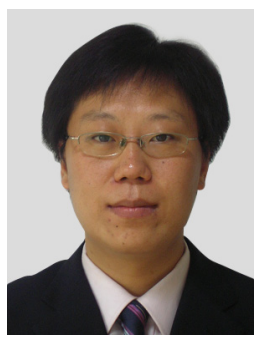

Celimuge Wu received the M.E. degree from Beijing Institute of Technology, Beijing, China, in 2006, and the PhD degree from the University of Electro-Communications, Tokyo, Japan, in 2010. Since 2010, he has been an assistant professor at the Graduate School of Information Systems, the University of ElectroCommunications. His current research interests include vehicular ad hoc networks, networking architectures and protocols.

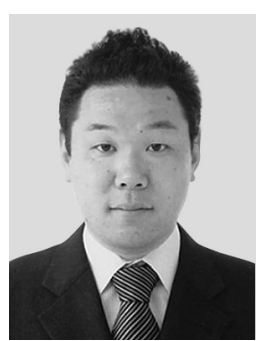

Satoshi Ohzahata received B.S., M.E., and D.E. degrees from the University of Tsukuba in 1998, 2000 and 2003, respectively. $\mathrm{He}$ was a Research Associate, Department of Computer, Information \& Communication Sciences at Tokyo University Agriculture and Technology from 2003-2007, and was an assistant professor of the same university from 2007-2009. Since 2009, he has been an associate professor at Graduate School of Information Systems, the University of Electro Communication. His interests are mobile ad hoc networks, the Internet architecture in mobile environments and Internet traffic measurement. He is a member of IEEE, ACM and IPSJ. 


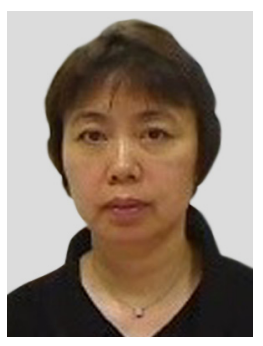

Yusheng Ji received B.E., M.E., and D.E. degrees in electrical engineering from the University of Tokyo. She joined the National Center for Science Information Systems, Japan (NACSIS) in 1990. Currently, she is a Professor at the National Institute of Informatics, Japan (NII), and the Graduate University for Advanced Studies (SOKENDAI). She is also appointed as a Visiting Professor at the University of Science and Technology of China (USTC). Her research interests include network architecture, resource management, and performance analysis for quality of service provisioning in wired and wireless communication networks. She is a member of IEEE, IEICE, and IPSJ.

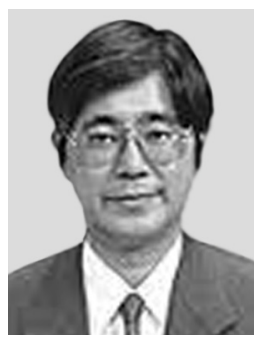

Toshihiko Kato received the B.E., M.E. and Dr. Eng. degrees electrical engineering from the University of Tokyo, in 1978, 1980 and 1983, respectively. He joined KDD in 1983 and worked in the field of communication protocols of OSI and Internet until 2002. From 1987 to 1988 , he was a visiting scientist at Carnegie Mellon University. He is now a professor of the Graduate School of Information Systems in the University of Electro-Communications in Tokyo, Japan. His current research interests include protocol for mobile Internet, high speed Internet and ad hoc network. 